\title{
Characterising Super-Earths
}

\author{
D. Valencia \\ ${ }^{1}$ Observatoire de la Cote d'Azur, BP 4229,06300 Nice, France [dianav@mit.edu]
}

* Now at the Earth, Atmospheric and Planetary Sciences Department, Massachusetts Institute of Technology, MA, 02139, USA

\begin{abstract}
The era of Super-Earths has formally begun with the detection of transiting low-mass exoplanets CoRoT-7b and GJ 1214b. In the path of characterising super-Earths, the first step is to infer their composition. While the discovery data for CoRoT-7b, in combination with the high atmospheric mass loss rate inferred from the high insolation, suggested that it was a rocky planet, the new proposed mass values have widened the possibilities. The combined mass range $1-10 M_{\oplus}$ allows for a volatile-rich (and requires it if the mass is less than $4 M_{\oplus}$ ), an Earth-like or a super-Mercury-like composition. In contrast, the radius of GJ 1214b is too large to admit a solid composition, thus it necessarily to have a substantial gas layer. Some evidence suggests that within this gas layer $\mathrm{H} / \mathrm{He}$ is a small but non-negligible component. These two planets are the first of many transiting low-mass exoplanets expected to be detected and they exemplify the limitations faced when inferring composition, which come from the degenerate character of the problem and the large error bars in the data.
\end{abstract}

\section{Introduction}

The discovery of low-mass transiting exoplanets CoRoT-7b and GJ1214b, marks the beginning of characterisation of super-Earths. Super-Earths are commonly defined as planets that are non-gaseous in nature. Meaning, they are either icy or ocean planets, depending on the equilibrium temperature, or rocky in composition. However, this physical definition is difficult to translate into a quantitative one because the threshold for forming gaseous planets in terms of mass (or size) depends on many parameters and thus, is model dependent. Furthermore, the current measured mass of a planet might have been greatly altered through atmospheric escape and other evolution processes after formation. In fact, CoRoT-7b and GJ 1214b exemplify this point: both planets have similar masses as reported in the discovery papers, but have very different radii, suggesting that CoRoT-7b is most likely a rocky planet, while GJ1214b is a miniNeptune.

The first step towards characterising super-Earths is to infer the composition from mass-radius measurements. Within this, the most important hurdle is to distinguish the planets that are solid from the ones that have a significant vapor-rich envelope, which translates into discriminating between the super-Earths and the mini-Neptunes. However, this is a challenge because the problem is degenerate in nature, as many dif- 
ferent compositions yield the same radius for a given mass. In this proceeding I discuss what we have learned from the two low-mass exoplanets in terms of their composition but mostly in terms of future prospects. I show how the new measurements for the mass of CoRoT-7b affect the inference of its composition.

\section{Interior Structure Models}

In general, interior structure models follow a standard prescription. They solve the structure equations (in differential or integral form) for density, mass, pressure, gravity, temperature or entropy. They treat the planet as the sum of layers of distinct composition (i.e. iron core, lower mantle, upper mantle, envelope of water) and tie the equations with an equation of state. Models that consider gaseous planets usually need to treat the evolution of the planet as it contracts due to the effect of cooling. For planets that are solid, the evolution can be disregarded as they cool very little, and the effect of temperature on the radius is negligible.

The results shown here are calculated with the model described in Valencia, O'Connell \& Sasselov 2006, and Valencia, Sasselov \& O'Connell 2007. for the solid planets (either terrestrial or icy). The results for the vapor planets or those with an envelope of $\mathrm{H} / \mathrm{He}$ are calculated by combining this model to that of Guillot \& Morel 2995, and Guillot 2005. The two models are connected at the surface/envelope interface to satisfy continuity in mass and pressure. See Valencia et al. 2010 for more details.

\section{CoRoT-7b}

Since the discovery paper and radial velocity (RV) follow-up on CoRoT-7b, both the reported radius $\left(R=1.68 \pm 0.09 R_{\oplus}\right.$, Leger et al. 2009$)$ and mass $\left(M=4.8 \pm 0.8 M_{\oplus}\right.$, Queloz et al. 2009) of this planet have changed. Thanks to improved parameters on the star, the size of this planet was reported to be smaller $R=1.58 \pm 0.10 R_{\oplus}$ (Bruntt et al. 2010). Recent studies that use different treatments of the RV data to better account for stellar activity have revised the mass to a larger value of $M=6.9 \pm 1.4 M_{\oplus}$ (Hatzes et al. 2010) and $M=8.5 \pm 1.5 M_{\oplus}$ (Ferraz-Mello et al. 2010), while confirming a second non-transiting planet. Also, Lanza et al. 2010 reports a false-alarm probability of less than $10^{-4}$ for two planets orbiting CoRoT-7. In contrast, a study by Pont, Aigrain \& Zucker 2010 that fits both stellar activity and uncertainties reports a lower statistical significance for CoRoT-7b with a mass estimation of only $M=1-4 M_{\oplus}$ and points to no compelling evidence for a second companion.

The new value for the radius and especially the new and different values for the mass have a dramatic effect on the interpretation of the composition of this planet. Figure 1 shows the mass-radius relations for different types of rocky compositions ranging from an iron-depleted case (i.e. a super-Moon) to an iron-pure planet. While both extremes are unlikely to exist for super-Earths, they show the bounds for a rocky composition. If the mass is less than $4 M_{\oplus}$, as suggested by Pont et al. 2010, the planet cannot be rocky in nature. It has to be a volatile-rich planet with an envelope of hydrogen and helium $(\mathrm{H} / \mathrm{He})$ or of $\mathrm{H}_{2} \mathrm{O}$-vapor. However, the size of the planet is not large enough to admit large amounts of $\mathrm{H} / \mathrm{He}$. Figure 2 shows the mass-radius relations for a planet with a $\mathrm{H} / \mathrm{He}$-envelope (red-family curves) overlaying an Earth-like nucleus. The maximum value for $\mathrm{H} / \mathrm{He}$ is a few parts in 10,000 by mass, which is very low and would be eroded in timescales of a few My given the insolation of the planet Valencia et al. 2010. With an estimated age of the system at 1.2-2.3 (Leger et al. 2010), H/He can be ruled out as being present in CoRoT-7b even for the low masses suggested by Pont 
et al. 2010. On the other hand, its size admits less than $30 \% \mathrm{H}_{2} \mathrm{O}$ by mass (figure 2), and the timescales for eroding this amount of water are a few Gy, compatible with the age of the system. Thus, if the mass is between $1-4 M_{\oplus}$, this planet is a $\mathrm{H}_{2} \mathrm{O}$-vapor planet.

On the other hand, the other two measurements for CoRoT-7b's radius suggest a planet denser than originally estimated, making the case for volatiles less plausible. From figures 1 and 2, a value of $M=6.9 \pm 1.4 M_{\oplus}$ corresponds to either an Earth-like composition or a vapor-planet with a low amount of $\mathrm{H}_{2} \mathrm{O}$ (less than $1 \%$ by mass). A value of $M=8.5 \pm 1.5 M_{\oplus}$ corresponds to a rocky composition that is denser than terrestrial - a super-Mercury (65\% iron core, $25 \%$ silicate mantle) fits the data well. Furthermore, this large density is hard to reconcile with the existence of a volatile envelope unless the nucleus is composed of iron-rich material. Thus, with the large uncertainty in the mass of CoRoT-7b, the only solid inference is that $\mathrm{H}-\mathrm{He}$ can be ruled out. It is also curious to notice that new estimates don't overlap with the original one, and barely overlap with each other, suggesting a combined mass range of $1-10 M_{\oplus}$.

\section{GJ 1214b}

The second transiting low-mass planet is GJ $1214 \mathrm{~b}$, with a mass of $6.55 M_{\oplus}$ and a radius of $2.7 R_{\oplus}$ this planet is not solid. Figure 3 shows the mass-radius relation for a $100 \%$ icy composition (dashed-grey line), and GJ 1214b's data lies well above it. From this simple observation, we can conclude that GJ 1214b has a gaseous envelope.

We model the structure of GJ $1214 \mathrm{~b}$ by considering as a boundary condition a temperature of $1000 \mathrm{~K}$ at 10 bars, following the results by Miller-Ricci-Kempton \& Fortney 2010. Figure 3 shows the results with mass-radius relations for a planet with different amounts of $\mathrm{H}_{2} \mathrm{O}$-vapor above a rocky interior, up to a composition of $100 \%$ water-vapor. They suggest that GJ $1214 \mathrm{~b}$ can be a pure water-vapor planet. However, this composition is unlikely to exist given that solids condense at much higher temperatures than water, thus some amount of solids is expected in the interior of GJ 1214b. If solids are present, then a lighter component needs to be present as well to account for the density of the planet. Therefore, $\mathrm{H} / \mathrm{He}$ is expected in the envelope of GJ 1214b. We find that the maximum amount of $\mathrm{H} / \mathrm{He}$ is $8 \%$ by mass, in agreement with Rogers \& Seager 2010. One caveat to our result is the assumption that the atmosphere's temperature at 10 bars is $1000 \mathrm{~K}$. We are currently relaxing this constraint to quantify the effect on the composition. To complicate the problem, the radius of GJ 1214b has also been recently revised. Carter et al. 2010 suggest that the radius of the planet may be smaller, making an argument for $\mathrm{H} / \mathrm{He}$ less compelling.

Interestingly, recent exciting observations of the spectra of GJ 1214b, albeit conflicting, seem promising in helping constrain the composition. While Croll et al. 2010 have suggested a $\mathrm{H} / \mathrm{He}$ atmosphere, Bean et al. 2010 claim a non-detection of $\mathrm{H} / \mathrm{He}$ that is explained by hazes or a water atmosphere.

\section{Discussion}

The problem of inferring composition of low-mass exoplanets is degenerate, so that even with perfect accuracy, just from mass and radius measurements alone it is not possible to uniquely determine the planet's composition (Valencia et al. 2007b). It is obvious that as the uncertainties in the data grow, the more ambiguous the problem is. However, obtaining accurate measurements has proven to be difficult and the case 


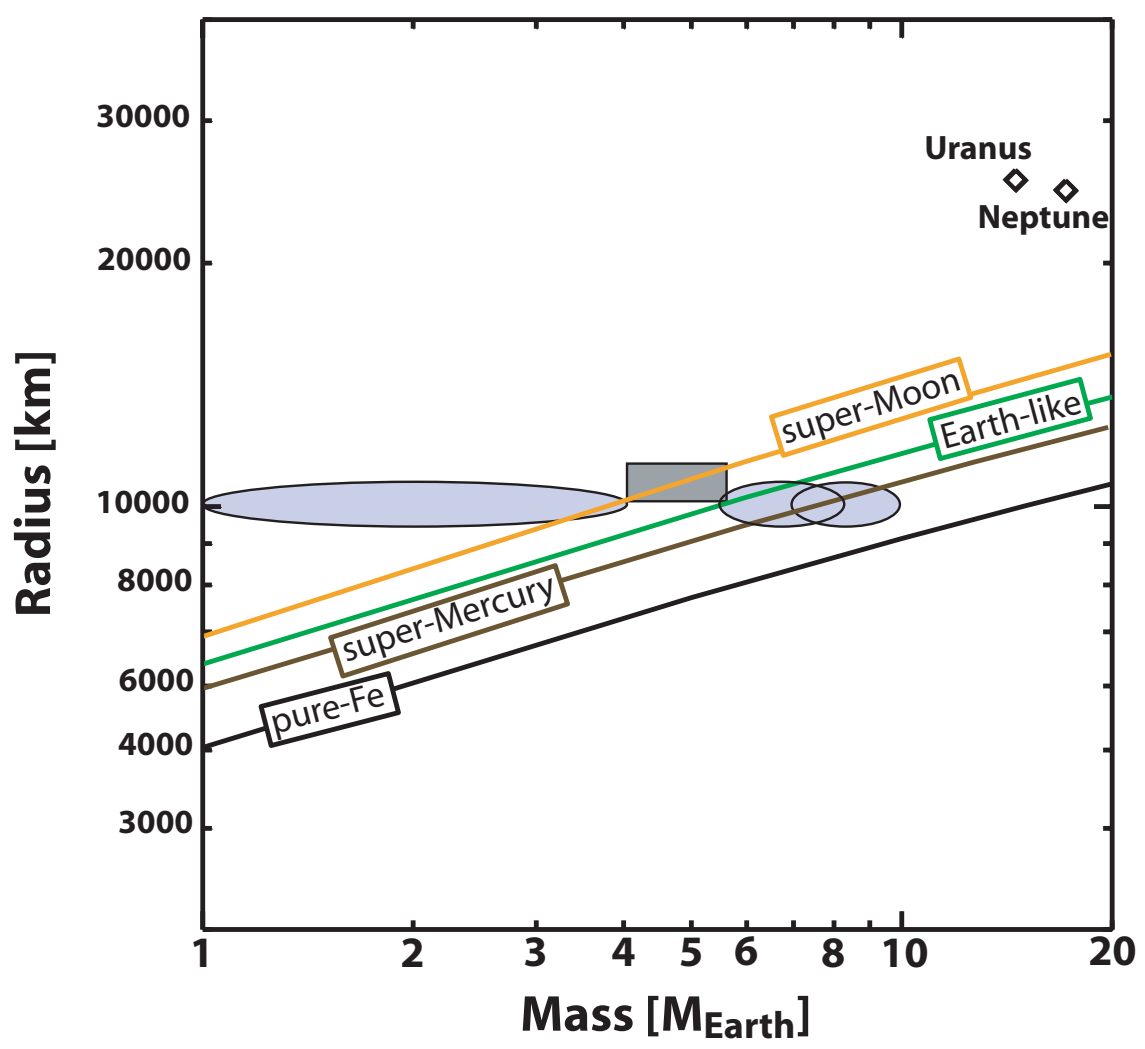

Figure 1.: Mass-Radius Relations for Rocky Planets. Different compositions are shown ranging from the lightest one, a super-Moon (with little or no iron), to a pure iron planet with increasing amounts of iron. An Earth-like planet corresponds to an iron core of $33 \%$ by mass below a silicate mantle of $67 \%$ by mass. A super-Mercury corresponds to an iron core of $65 \%$ by mass below a silicate mantle of $65 \%$ by mass. The original value for the mass and radius of CoRoT- $7 b$ is shown in a grey box. The newest estimations are shown in ellipses that correspond to a $1-\sigma$ range according to the results by Pont et al. 2010 (left ellipse), Hatzes et al. 2010 (middle ellipse) and Ferraz-Mello et al. 2010 (right ellipse).

of CoRoT-7b exemplifies well the complications of determining the mass of a planet around an active star.

With the revised values for the mass, the composition of this planet went from being rocky and depleted in iron with respect to Earth, to being either a small vapor planet or a rocky one with more iron than Earth. Even if we only consider the uncertainty added by the studies of Hatzes et al. 2010 and Ferraz-Mello et al. 2010, which both point towards a rocky composition, there is much to be gained from distinguishing between the two. The measurement of Hatzes et al. 2010 points towards a more Earth-like composition, while that of Ferraz-Mello et al. 2010 suggests a more Mercury-like composition. Discerning the two would test the validity of assuming similar refractory ratios between the parent star and its planets. Even in our solar system this is an open question. While Earth's refractory ratios are largely in agreement with 
Detection and Dynamics of Transiting Exoplanets

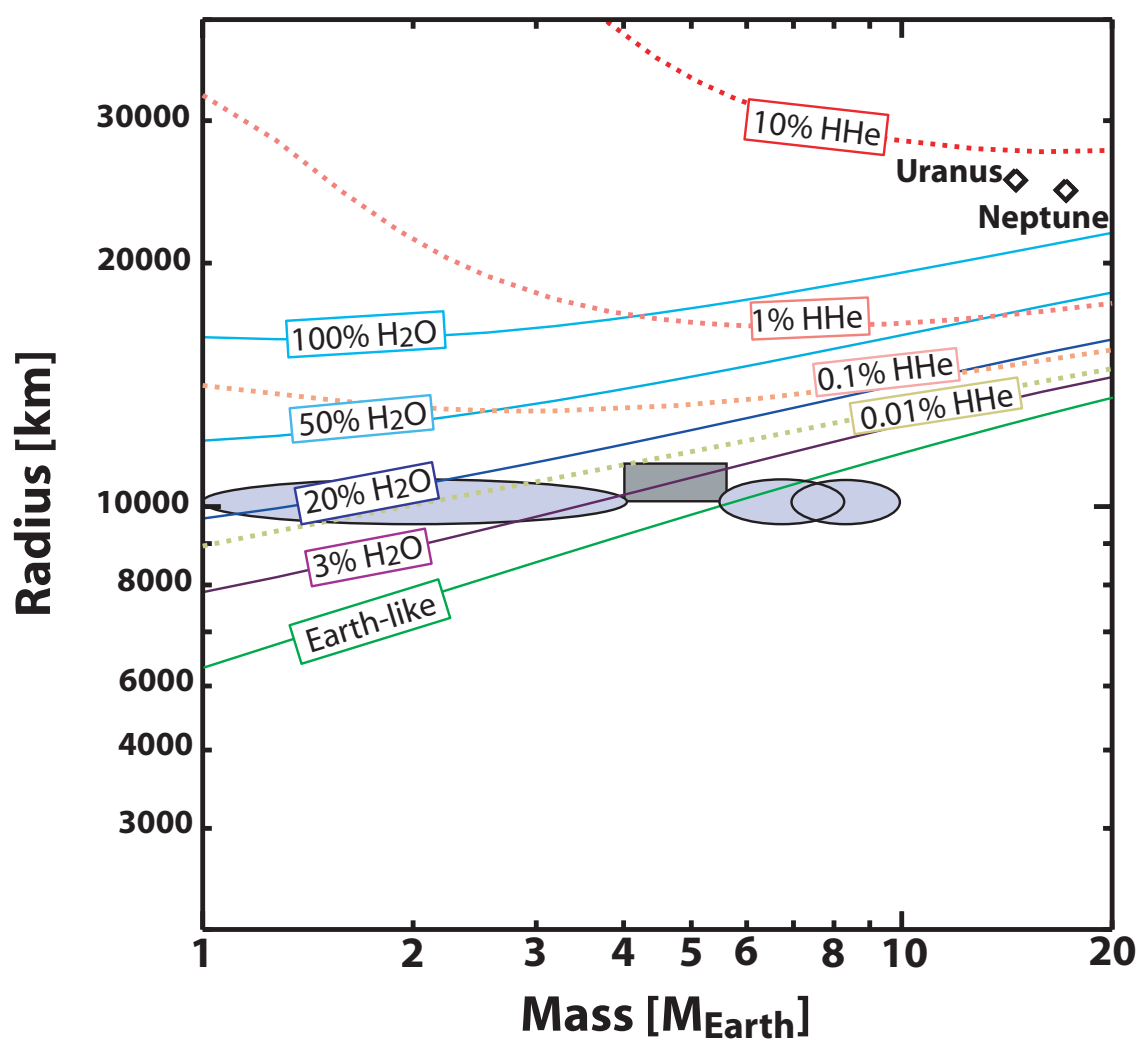

Figure 2.: Mass-Radius Relations for Fluid Planets. Different lines show different compositions: the blue family corresponds to planets with $\mathrm{H}_{2} \mathrm{O}$-vapor envelope above an earth-like nucleus in amounts of 3, 20, 50 and 100\% $\mathrm{H}_{2} \mathrm{O}$ by mass; the red family corresponds to planets with a H-He envelope above an Earth-like nucleus in amounts of $0.01,0.1,1$, and 10\% by mass. The original value for the mass and radius of CoRoT-7b is shown in a grey box. The newest estimations are shown in ellipses (see caption in figure 1 for details).

carbonaceous chondrites (McDonough \& Sun 95), Mars' ratios are less well determined (Ohtani \& Kamaya 1992), and Mercury's composition is known to be enriched in iron with a possible explanation coming from stripping of the mantle by a giant impact. One advantage that super-Earths offer is that they seem to not be as susceptible to compositional alteration from impacts (Marcus et al. 2010, as the small planets, and thus, might better track planetesimal composition.

Any efforts to better constrain the mass of CoRoT-7b, such as simultaneously observing the planet in transit and in radial velocity, are worth pursuing. It is important to determine if this is the first exoplanet with an Earth-like composition (in terms of the major elements), and to lay out the necessary steps to characterise low-mass planets around active stars.

On the other hand, it is easy to conclude that GJ 1214b has a gaseous envelope, since it is too big to be a solid planet. However, the interesting question is to determine the nature of its gas layer, and in particular if hydrogen and helium are present. Ac- 


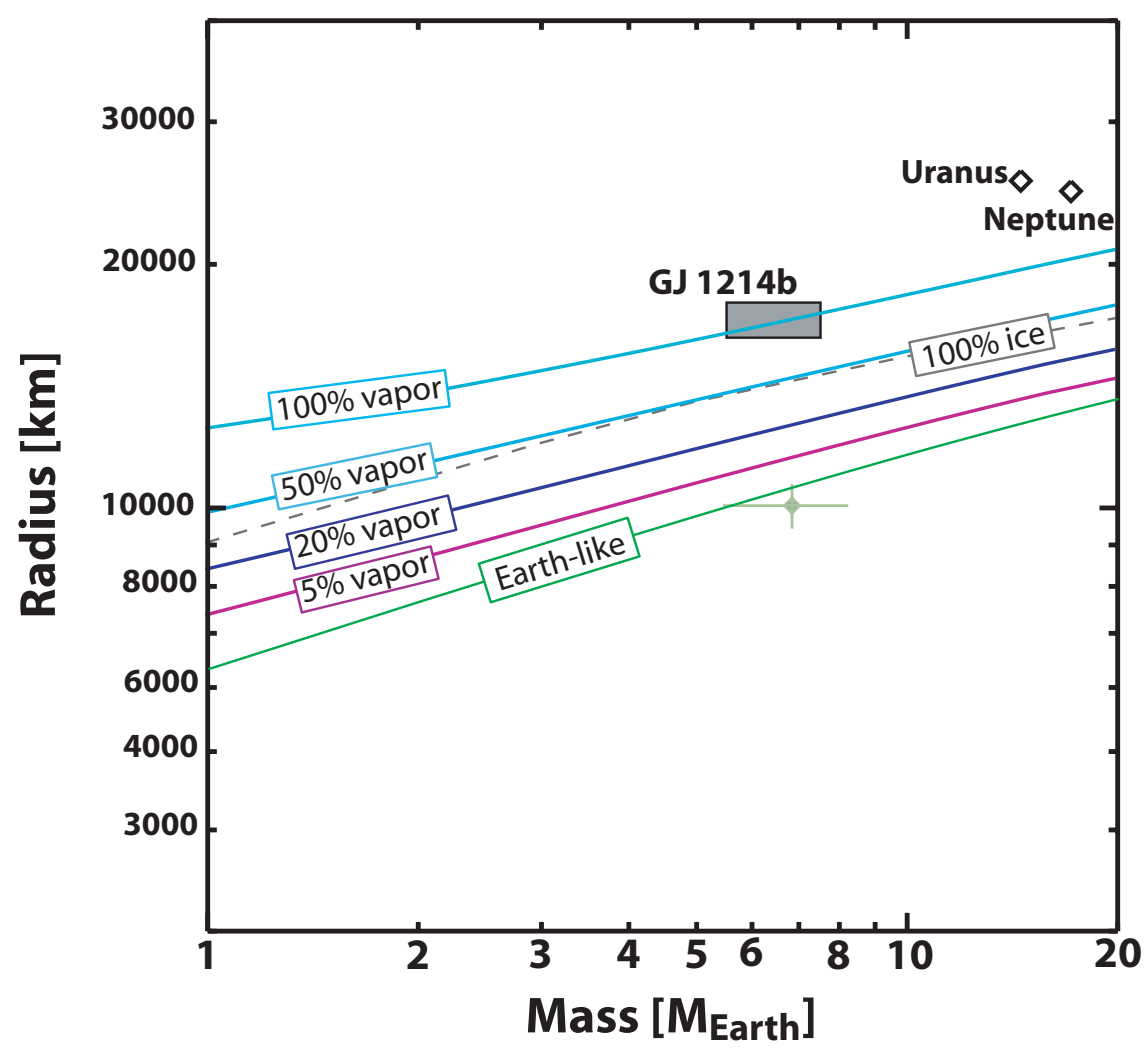

Figure 3.: Mass-Radius Relations for GJ 1214b. We consider planets with an $\mathrm{H}_{2} \mathrm{O}$-vapor envelope above an earth-like nucleus in amounts of 5, 20, 50 and 100\% $\mathrm{H}_{2} \mathrm{O}$ by mass. These relationships were calculated with a boundary condition of $1000 \mathrm{~K}$ at 10 bars (following Miller-Ricci and Fortney 2010). The dashed-grey line shows the relationship for an icy composition, and the grey box shows the measured mass of GJ $1214 b$.

cording to our models and the radius proposed by Charbonneau et al. 2009, there has to be a lighter component than $\mathrm{H}_{2} \mathrm{O}$, with the obvious candidate being a $\mathrm{H} / \mathrm{He}$ mixture ${ }^{1}$. Its amount will depend on the amount of refractory material the planet harbors. However, this result depends on the boundary condition we applied for the atmosphere. If the planet is cooler than $1000 \mathrm{~K}$ at 10 bars, the upper limit for $\mathrm{H} / \mathrm{He}$ increases, and conversely, if the planet is hotter, there might not be a need for a component lighter than $\mathrm{H}_{2} \mathrm{O}$. This is also the case, if the radius is indeed as small as suggested by Carter et al. 2010. Interestingly, in the near future observations of GJ 1214b's spectra will paint a clearer picture about the composition of the very top of the atmosphere (a few millibars), which then needs to be tied to the composition of the deeper portions of the envelope.

\footnotetext{
${ }^{1}$ Rogers \& Seager 2010 have also suggested a $\mathrm{H}_{2}$ dominated atmosphere.
} 
CoRoT-7b and GJ 1214b are the first two of many low-mass exoplanets expected in the future to have measured masses and radii. They exemplify the challenges that observers face in determining these parameters correctly (i.e. activity of the star, validity of stellar models, etc), as well those by theorists in inferring composition given the degenerate character of the problem. From a theorist perspective, it is obvious that the smaller the error bars the better, but to break the degeneracy other sources of information, such as constraints from atmospheric escape and formation scenarios are key.

\section{References}

Bean, J., Miller-Ricci Kempton, E., Homeier, D. 2010, Nature, 468, 669

Bruntt, H., Deleuil, M., Fridlund, M., et al. 2010, A\&A, in press

Carter, B., Winn, J., Holman, M., et al. 2010. ApJ, submitted

Charbonneau, D., Berta, Z., Irwin, J., et al. 2009, Nature, 462, 891

Croll, B, Albert, L, Jayawardhana, R., et al. 2010, presentation at IAU Symp. 276

Ferraz-Mello, S., Tadeu dos Santos, M., Beauge, C., et al. 2010, A\&A, submitted Guillot, T., \& Morel, P. 1995, A\&AS, 109, 109

Guillot, T. 2005, Annual Review of Earth and Planetary Sciences, 33, 493

Hatzes, A. Dvorak, R., Wuchterl G., et al. A\&A, 520, A93

Lanza, A., Bonomo, A., Moutou, C., et al. 2010, A\&A, 520, A53

Leger, A., Rouan, D., Schneider, R. et al. 2009, A\&A, 506, 287

Marcus, Rl, Sasselov, D., Hernquist, L. \& Stewart, S. 2010, ApJL, 712, L73

McDonough, W. F. \& S.-s. Sun. 1995, Chem. Geol., 120, 223

Miller-Ricci, E., \& Fortney, J., 2010, ApJL, 716, L74

D. Queloz, F. Bouchy, C. Motou, et al. 2009, A\&A, 506, 303

Ohtani, E., N. Kamaya. 1992. GRL, 19, 2239

Pont, F., Aigrain, S., \& Zucker, S. 2010, MNRAS, in press

Rogers, L., \& Seager, S. 2010, ApJ, 716, 1208

Valencia, D., O'Connell, R. J., \& Sasselov, D. D. 2006, Icarus, 181, 545

Valencia, D., Sasselov, D. D., \& O'Connell, R. J. 2007, ApJ, 656, 545

Valencia, D., Sasselov, D. D., \& O'Connell, R. J. 2007, ApJ, 665, 1413

Valencia, D., Ikoma, M., Guillot, T., \& Nettelmann, N. 2010, A\&A 516, A20 